\title{
Macrófitas aquáticas de ilhas flutuantes (baceiros) nas sub-regiões do Abobral e Miranda, Pantanal, MS, Brasil ${ }^{1}$
}

\author{
Marco Otávio Pivari ${ }^{2,4}$, Vali Joana Pott ${ }^{3}$ e Arnildo Pott ${ }^{3}$
}

Recebido em 21/12/2006. Aceito em 13/08/2007

\begin{abstract}
RESUMO - (Macrófitas aquáticas de ilhas flutuantes (baceiros) nas sub-regiões do Abobral e Miranda, Pantanal, MS, Brasil). A associação entre as formas biológicas flutuante e epífita, relacionadas a macrófitas aquáticas, constitui o que se chama genericamente de ilha flutuante. Esta denominação é aplicada ao estabelecimento de uma ou mais epífitas sobre indivíduos vivos de espécies flutuantes, como sinônimo de camalotal ou, ainda, para se referir à vegetação flutuante que se desenvolve sobre um solo orgânico em decomposição (histossolo). Realizou-se o levantamento das espécies presentes em cinco baceiros nas sub-regiões pantaneiras do Abobral e Miranda e o estabelecimento das similaridades florísticas entre estes, a fim de evidenciar aspectos da dinâmica sucessional de ilhas flutuantes. Foram encontradas 66 espécies de macrófitas aquáticas epífitas, distribuídas em 27 famílias, sendo Cyperaceae e Poaceae as mais representativas. O número de espécies por baceiro variou de 7 a 39, sendo os táxons comuns a todos esses Ludwigia leptocarpa (Nutt.) H. Hara e Oxycaryum cubense (Poepp. \& Kunth) Palla, ao nível específico, e Asteraceae, Cyperaceae, Onagraceae, Poaceae e Polygonaceae, com relação às famílias. Outras formas biológicas, além da epífita, foram encontradas associadas aos baceiros, ou ainda, se mostraram adaptadas às condições do histossolo, podendo ser consideradas "epífitas casuais". Baceiros em estágios sucessionais mais próximos apresentam maior similaridade florística e, no geral, quanto mais avançada a sucessão natural, maior a riqueza específica.
\end{abstract}

Palavras-chave: plantas aquáticas, florística, sucessão aquática, ilha flutuante, Pantanal

\begin{abstract}
Aquatic macrophytes of floating mats ("baceiros") in the Abobral and Miranda Pantanal Wetlands, Mato Grosso do Sul State, Brazil). The association between floating and epiphytic biological forms, regarding aquatic macrophytes, creates what are called floating mats. This concept refers to the establishment of one or more epiphytes on live individuals of floating species, as a synonym of "camalotal," or applied to floating vegetation that grows on organic soil in decomposition (histosol). A checklist of species was made of five floating mats in the Abobral and Miranda Pantanal Wetlands and floristic similarities among these mats were calculated to show aspects of sucessional dynamics on floating mats. A total of 66 species of epiphytic aquatic macrophytes were found, distributed in 27 families, Cyperaceae and Poaceae being the most representative. The number of species per mat varied from 7 and 39 , with some taxa common to all mats, such as Ludwigia leptocarpa (Nutt.) H. Hara and Oxycaryum cubense (Poepp. \& Kunth) Palla, at the specific level, and Asteraceae, Cyperaceae, Onagraceae, Poaceae and Polygonaceae, at the family level. Other biological forms besides epiphytes were found associated with "baceiros" or adapted to histosol conditions, being considered "casual epiphytes". "Baceiros" in similar successional stages of development have high floristic similarity, and in general, the more advanced the natural succession, the higher the number of species.
\end{abstract}

Key words: aquatic plants, floristics, aquatic succession, floating mat, wetland

\section{Introdução}

Macrófita aquática, segundo Esteves (1998), é um termo já consagrado na literatura científica internacional, o qual se aplica aos vegetais que ocorrem em ambientes úmidos, de uma forma geral, não levando em consideração aspectos taxonômicos nesse agrupamento. Essa definição foi proposta por Cook (1974), se referindo "aos vegetais vasculares cujas partes fotossinteticamente ativas estão permanentemente, ou por alguns meses, submersas ou flutuantes em água e são visíveis a olho nu". Todavia, outras terminologias, como hidrófita (Martins \& Carauta 1984), frequientemente são utilizadas.

Segundo as classificações sobre formas biológicas aplicadas às plantas aquáticas, propostas por Irgang et al. (1984) e Pedralli (2000), epífitas são aquelas espécies que se estabelecem e se desenvolvem sobre

\footnotetext{
1 Parte da Dissertação de Mestrado do primeiro Autor

2 Universidade Federal de Mato Grosso do Sul, Centro de Ciências Biológicas e da Saúde, Departamento de Biologia, Cidade Universitária s.n., 79070-900 Campo Grande, MS, Brasil

3 Empresa Brasileira de Pesquisa Agropecuária, Embrapa Gado de Corte, Rodovia BR 262, Km 4, Vila Popular, Campo Grande, MS, Brasil

4 Autor para correspondência: marcopivari@yahoo.com.br
} 
indivíduos de espécies flutuantes livres ou fixas. Dessa maneira, são consideradas macrófitas aquáticas, pois mantém as raízes em contato direto e permanente com a água, vindo a morrer caso estas fiquem expostas ao ambiente seco. O primeiro caso de epifitismo aquático foi descrito por Tur (1965), que observou o estabelecimento de Oxycaryum cubense sobre Salvinia minima, em trabalho realizado no Rio Paraná.

A associação dessas duas formas biológicas, flutuantes e epífitas, além do solo orgânico que elas produzem, constitui o que se chama genericamente de ilha flutuante (Cuadrado \& Neiff 1993). No Pantanal brasileiro, essa denominação é aplicada em várias ocasiões, como: na associação entre uma ou mais epífitas e indivíduos vivos de espécies flutuantes; como sinônimo de camalotal, que identifica especificamente a ocorrência de epífitas sobre indivíduos de espécies de Eichhornia; ou ainda, para se referir à vegetação que se desenvolve sobre um solo orgânico, de natureza flutuante.

De acordo com o estágio sucessional das ilhas flutuantes, estas apresentam diferentes características, como, por exemplo, o tipo de substrato ou sua composição florística. Existem, regionalmente, diferentes denominações para os estágios das ilhas flutuantes (Pott \& Pott 2000). "Camalote" ou "camalotal" (Lima et al. 1999) são termos empregados para ilhas flutuantes recém formadas, onde indivíduos de Eichhornia compõem o substrato flutuante, ainda não tendo sido "substituídos" por solo orgânico. "Embalsado" (Tur 1972), "batume" (Silva 1984) ou "baceiro" (Pott \& Pott 2000) se aplicam às ilhas em estágios de sucessão mais avançados, que apresentam solo orgânico como substrato flutuante de fixação para espécies epífitas. Em todas as situações, encontra-se a presença de macrófitas aquáticas epífitas sobre um substrato flutuante, seja este constituído por indivíduos vivos, ou por matéria orgânica morta. O que determina a característica do substrato é o tempo de sucessão natural da ilha flutuante (Pott \& Pott 2003). Sendo assim, ilhas flutuantes em estágios sucessionais iniciais ainda apresentam a base flutuante composta por macrófitas vivas, enquanto em ilhas mais antigas houve tempo necessário para o desenvolvimento de um solo orgânico flutuante.

Lima et al. (1999) descreveram a formação do solo orgânico de ilhas flutuantes da seguinte forma: "o crescimento e expansão de macrófitas aquáticas em áreas inundáveis e permanentemente alagadas, podem culminar na formação de extensos tapetes verdes, ricos em matéria orgânica proveniente da decomposição de macrófitas, que se acumula nas raízes e estolões das mesmas, iniciando a formação de uma camada flutuante. Esta aumenta à medida que ocorrem novas deposições, favorecendo a implantação e estabelecimento de plantas pantanosas". Com a retenção contínua de restos vegetais, ocorre um engrossamento desta camada flutuante, a qual pode alcançar vários metros de profundidade, fornecendo assim maior capacidade de suporte, servindo de substrato para arbustos e árvores (Silva 1984). Segundo Neiff \& Orellana (1980 apud Neiff 1982), tal substrato denomina-se histossolo por ser constituído apenas por tecidos vegetais e apresentar pouca quantidade (menos de 30\%) de componentes mineralógicos de solo terrestre.

Segundo Neiff (1982), "embalsados" diferem dos camalotes por possuírem maior capacidade de suporte mecânico, derivado da presença de solo orgânico (histossolo); maior complexidade da vegetação (com maior riqueza específica e bioformas, maior número de estratos, maior coerência, etc.); gravitação na coluna d'água em função de sua cobertura e tempo de permanência; e por representarem fases mais avançadas da sucessão vegetal. Segundo o mesmo autor, a composição florística dos "embalsados" assemelha-se à vegetação de áreas de turfeiras, as quais apresentam um horizonte orgânico bem desenvolvido no solo.

Essas formações também poderiam ser consideradas ecótonos por constituírem elementos de transição entre sistemas ecológicos adjacentes, ou, mais tradicionalmente, entre tipos de vegetação (Risser 1995). Nesse contexto, a ocorrência de baceiros caracteriza uma dessas áreas, já que estes se encontram entre os limites dos ecossistemas aquático e terrestre, além de apresentar composição florística formada por espécies comuns a áreas pantanosas ou de brejos, ou seja, por vegetação com características mistas de ambientes aquáticos e de terras não alagáveis.

As ilhas flutuantes permanecem nos diferentes corpos d'água livremente, trocando constantemente de posição, conforme as forças do vento e correnteza (Tur 1972). Podem, eventualmente, ancorar nas margens desses, especialmente em secas pronunciadas (Pott \& Pott 2003), possibilitando sua morte e oxidação do solo orgânico, o qual pode ser atingido pelo fogo, liberando nutrientes.

No caso do Pantanal sul-mato-grossense, a vegetação aquática, em especial de ilhas flutuantes, está relacionada a vários fatores sócio-econômicos, como a utilização do solo orgânico proveniente dessas, para o 
cultivo de hortaliças, e por abrigar iscas (Tuvira - Gymnotus carapo, Caranguejo - Dilocarcinus paguei paguei e Caramujos - espécies de Pomacea) que são destinadas ao comércio pesqueiro. Tais ilhas também assumem um papel ecológico primordial, por proporcionarem condições favoráveis à nidificação de aves e jacarés, além de fornecerem alimento e abrigo a peixes e outros organismos aquáticos (Pott \& Pott 2003).

Contudo, trabalhos a respeito desse tipo de vegetação são escassos no Brasil, sobretudo no Pantanal. O objetivo desta pesquisa foi determinar a composição florística de baceiros nas sub-regiões pantaneiras do Abobral e Miranda, e identificar similaridades florísticas entre esses, fornecendo assim subsídios para o entendimento da dinâmica sucessional de ilhas flutuantes.

\section{Material e métodos}

O Pantanal apresenta clima tropical sub-úmido do tipo Aw (Köppen 1931), com média pluviométrica de $1.100 \mathrm{~mm}$ anuais e temperatura média anual de $26^{\circ} \mathrm{C}$. Caracteriza-se por duas estações definidas, sendo uma chuvosa (outubro a março) e uma relativamente seca (abril a setembro), havendo defasagem entre o clima (chuvas) e a umidade no solo, tanto maior quanto mais deprimida e quanto mais afetada por inundação fluvial for a área (Wilhelmy 1957).

Toda planície pantaneira, de origem sedimentar aluvial quaternária (holocênica), é parcial e periodicamente alagável em função da sua baixíssima declividade, que em longas áreas atinge $2-5 \mathrm{~cm} / \mathrm{km}$ de norte a sul e $30-50 \mathrm{~cm} / \mathrm{km}$ de leste a oeste (Brasil 1979). Assim, são carreados anualmente sedimentos aluviais, que obstruem o sistema de drenagem, propiciando a formação de um ambiente com características próprias (Amaral Filho 1986), submetido a pulsos de inundação (Junk \& Silva 1999). As áreas alagadas correspondem aos alagados, rios, corixos, baías, lagoas temporárias, vazantes e caixas de empréstimo (Pott \& Pott 2000), estas construídas pelo homem.

Devido à diversidade de ambientes e à grande amplitude territorial, o Pantanal brasileiro é subdividido em sub-regiões conforme suas características particulares, como influência de rios, tipo de solo, relevo, fitofisionomias, dentre outras. Segundo Silva \& Abdon (1998), o Pantanal brasileiro apresenta onze subdivisões, cobrindo parte dos Estados de Mato Grosso e Mato Grosso do Sul. As sub-regiões do Abobral e Miranda destacam-se economicamente devido às atividades de pecuária, ecoturismo e por fornecer itens de subsistência para a população pantaneira, como iscas coletadas para o comércio da pesca. Ocupam uma faixa do oeste de Mato Grosso do Sul, apresentando solos mistos compostos por areia e argila, onde predominam campos alagáveis cobertos por pastagens nativas, entremeados por capões e cordilheiras (mata estacional semi-decídua) (Allem \& Valls 1987). Eventualmente são encontrados baceiros nessas sub-regiões, especialmente em corixos e baías.

O trabalho foi realizado de março/2005 a março/2006, abrangendo dois períodos estacionais, um de chuva e um de seca, totalizando um ciclo anual de inundação e vazante pantaneira, ou ainda, período relativo a um pulso de inundação. Para isso, contou com expedições de campo bimensais, com duração de três dias cada, através de embarcações e percursos a pé. Cinco baceiros de diferentes áreas, três presentes em braços abandonados do Rio Vermelho: I $\left(19^{\circ} 36^{\prime} 47^{\prime \prime} \mathrm{S}\right.$; $\left.56^{\circ} 58^{\prime} 35^{\prime \prime} \mathrm{W}\right)$ a $97 \mathrm{~m}$ de altitude, II $\left(19^{\circ} 37^{\prime} 15^{\prime \prime} \mathrm{S} ; 56^{\circ} 57^{\prime} 59^{\prime \prime} \mathrm{W}\right)$ a $97 \mathrm{~m}$ de altitude, e III $\left(19^{\circ} 37^{\prime} 13^{\prime \prime} \mathrm{S} ; 56^{\circ} 58^{\prime} 08^{\prime \prime} \mathrm{W}\right)$ a $99 \mathrm{~m}$ de altitude; e dois

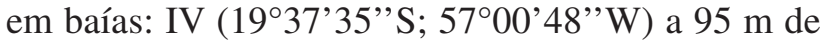

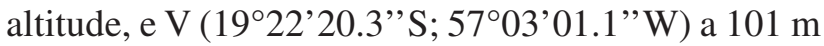
de altitude, foram estudados no presente trabalho. Localizam-se nas sub-regiões de Miranda (I a IV) e Abobral (V), oeste do Estado de Mato Grosso do Sul.

Os vegetais constituintes de ilhas flutuantes, que, segundo as definições de formas biológicas propostas por Irgang et al. (1984) e Pedralli (2000) são considerados epífitos, foram inventariados. Além destes, indivíduos classificados como flutuantes livres, flutuantes fixos ou submersos fixos também foram amostrados, a fim de complementar o entendimento a respeito da formação de ilhas flutuantes, uma vez que contribuem de fato com a deposição de camadas vegetais no histossolo do baceiro após sua morte.

Representantes de todas as espécies em estado reprodutivo (esporos, flor e/ou fruto) foram coletados e processados segundo técnicas de herborização, e, posteriormente, incluídos aos acervos dos herbários HMS localizado na Embrapa Gado de Corte e CGMS, campus da Universidade Federal de Mato Grosso do Sul. Tais técnicas consistem na prensagem, secagem em estufa e montagem das exsicatas. A identificação das espécies foi feita através de comparação morfológica e consulta à bibliografia especializada (Pott $\&$ Pott 2000). Por fim, duplicatas do material coletado foram enviadas aos especialistas de diversas famílias botânicas para confirmação das identificações. 
O inventário das espécies foi elaborado segundo o sistema de classificação APG II - Angiosperm Phylogeny Group (2003), para angiospermas, e de Kramer \& Green (1990) para pteridófitas, acrescido pelos nomes vulgares regionais e informações de ocorrência por baceiro pesquisado. A terminologia nomenclatural e abreviações dos nomes dos autores seguiram Brummitt \& Powell (1992). O estudo da similaridade florística entre as áreas (baceiros) foi realizado segundo aplicação do índice de Sørensen (1948) e de análise de Cluster. Esta foi estabelecida de acordo com a distância euclidiana simples obtida por matriz de presença/ausência de espécies, através do software estatístico SPSS 13.0, e representada graficamente por meio de dendrograma.

\section{Resultados e discussão}

O levantamento florístico das áreas estudadas revelou a presença de 66 espécies de macrófitas aquáticas inseridas na forma biológica epífita. Estas se distribuem em 27 famílias botânicas e são agrupadas em 48 gêneros (Tab. 1). Apenas duas espécies, Ludwigia leptocarpa e Oxycaryum cubense, estiveram presentes nos cinco baceiros, enquanto as famílias comuns a todos esses foram Asteraceae, Cyperaceae, Onagraceae, Poaceae e Polygonaceae.

O número de espécies epífitas referentes aos cinco baceiros amostrados nas sub-regiões do Abobral e Miranda é superior ao encontrado em levantamentos já realizados para esse tipo de vegetação. Pott \& Pott (2003) observaram apenas nove espécies para a subregião da Nhecolândia, enquanto Lima et al. (1999) citaram 19 para a Baía Piuvial, localizada na sub-região de Poconé. Em estudos realizados no vale de inundação do Rio Paraná, correspondente ao Chaco Argentino, Neiff (1982) verificou o estabelecimento de 24 espécies de macrófitas epífitas, enquanto Tur (1972) relatou 54 espécies relacionadas a ilhas flutuantes, na região do Médio Paraná, sendo que apenas uma parcela deste número corresponde às hidrófitas dessa forma biológica.

As famílias mais numerosas foram Cyperaceae e Poaceae, apresentando nove espécies cada, o que representa juntas, $27,3 \%$ das macrófitas aquáticas epífitas amostradas. Na seqüência destacaram-se Asteraceae, Onagraceae Fabaceae e Polygonaceae, com seis, cinco, quatro e quatro espécies, respectivamente (Fig. 1). Os gêneros com maior número de espécies encontrados foram Ludwigia (Onagraceae) - cinco espécies; Cyperus (Cyperaceae) e Polygonum
(Polygonaceae) - quatro espécies cada. Apenas uma família, Pteridaceae, está incluída em Pteridophyta, enquanto as demais se distribuem dentro de Angiospemae.

O número de espécies entre os cinco baceiros pesquisados variou de 39 a sete espécies. Tal variação pode ser atribuída aos estágios sucessionais das ilhas flutuantes, pois quanto maior o tempo de sucessão da vegetação, maior a complexidade desta em vários aspectos, sendo um destes, a riqueza específica (Neiff 1982). Dessa forma, os Baceiros 5 e 2, localizados na Baía Boa Sorte e no Rio Vermelho, apresentaram maior diversidade de espécies, 39 e 36, respectivamente. Contrariamente, os Baceiros 1 e 3, ambos localizados no Rio Vermelho, destacaram-se com menor número de espécies: sete e dez, constituindo as ilhas em estágios mais iniciais, dentre as analisadas no trabalho. Foram registradas no Baceiro 4, presente na Baía da Medalha, 18 espécies, devido ao estágio intermediário de sucessão natural desse, quando comparado aos demais.

A espessura do solo orgânico dos baceiros fornece outro indício da etapa de sucessão em que os mesmos se encontram, pois quanto maior o tempo de permanência da vegetação na coluna d'água, maior a quantidade de tecido morto depositado na camada flutuante. Sendo assim, o Baceiro 5 destacou-se com maior espessura média do histossolo dentre as ilhas flutuantes estudadas $(64 \mathrm{~cm})$, seguido pelos Baceiros 2, 4, 1 e $3(35 \mathrm{~cm}, 24 \mathrm{~cm}, 22 \mathrm{~cm}$ e $9 \mathrm{~cm}$, respectivamente), concordando com os diferentes estágios de sucessão apontados pela diversidade de espécies.

Segundo Pott \& Pott (2003), as ilhas flutuantes apresentam espécies características de acordo com o estágio da sucessão observada. Tal estudo foi corroborado pelos resultados deste trabalho, já que espécies como Eleocharis plicarhachis (referida como E. mutata em Pott \& Pott 2000; Pott \& Pott 2003), Ludwigia nervosa, Imperata tenuis, Luziola bahiensis e espécies de Mikania, esperadas em baceiros mais antigos, foram observadas no baceiro 5. Esse fato reforça a idéia desta ilha flutuante representar o estágio sucessional mais avançado, dentre as cinco comunidades abordadas. O mesmo esquema proposto por esses autores demonstra, por outro lado, que espécies de Salvinia, Eichhornia azurea, Limnobium laevigatum, Utricularia gibba e Pistia stratiotes são encontradas em estágios iniciais de formação de baceiros. De fato, todos esses táxons foram observados entre os indivíduos epífitos do Baceiro 3, o que permite inferir, juntamente com outras 
Tabela 1. Inventário das macrófitas aquáticas epífitas, presentes em cinco baceiros no Pantanal, sub-regiões do Abobral e Miranda, município de Corumbá, MS, Brasil (período março/2005-fevereiro/2006).

Família/Espécie

Nome vulgar

Baceiro

\begin{tabular}{ccccc}
\multicolumn{5}{c}{ Baceiro } \\
\hline 1 & 2 & 3 & 4 & 5
\end{tabular}

ACANTHACEAE

Justicia laevilinguis (Nees) Lind.

ALISMATACEAE

Echinodorus lanceolatus Rataj

E. paniculatus Micheli

APOCYNACEAE

Metastelma berterianum (Spreng.) Decne.

Rhabdadenia pohlii Müll. Arg.

ARALIACEAE

Hydrocotyle ranunculoides L.f.

ASTERACEAE

Baccharis medullosa DC.

Enydra radicans (Willd.) Lack

Erechtites hieraciifolius (L.) Raf. ex DC.

Eupatorium candolleanum Hook. \& Arn.

Mikania sp.

Pacourina edulis Aubl.

COMMELINACEAE

Commelina schomburgkiana Klotzsch

CONVOLVULACEAE

Ipomoea chiliantha Hallier $\mathrm{f}$.

I. rubens Choisy

I. subrevoluta Choisy

CUCURBITACEAE

Cayaponia podantha Cogn.

CYPERACEAE

Cyperus gardneri Nees

C. haspan $\mathrm{L}$.

C. odoratus $\mathrm{L}$.

Cyperus sp.

Eleocharis minima Kunth

E. plicarhachis (Griseb.) Svenson

Fimbristylis sp.

Lipocarpha sphacelata (Vahl) Kunth

Oxycaryum cubense (Poepp. \& Kunth) Palla

EUPHORBIACEAE

Caperonia castaneifolia (L.) A. St.-Hil.

FABACEAE

Aeschynomene sensitiva Sw.

Mimosa sp.

Sesbania virgata (Cav.) Pers.

Vigna lasiocarpa (Mart. ex Benth.) Verdc.

HYDROCHARITACEAE

Limnobium laevigatum (Humb. \& Bonpl. ex Willd.) Heine

HYDROLEACEAE

Hydrolea spinosa $\mathrm{L}$.

LAMIACEAE

Hyptis brevipes Poit.

LENTIBULARIACEAE

Utricularia gibba L.

MALVACEAE

Hibiscus sororius L. f.

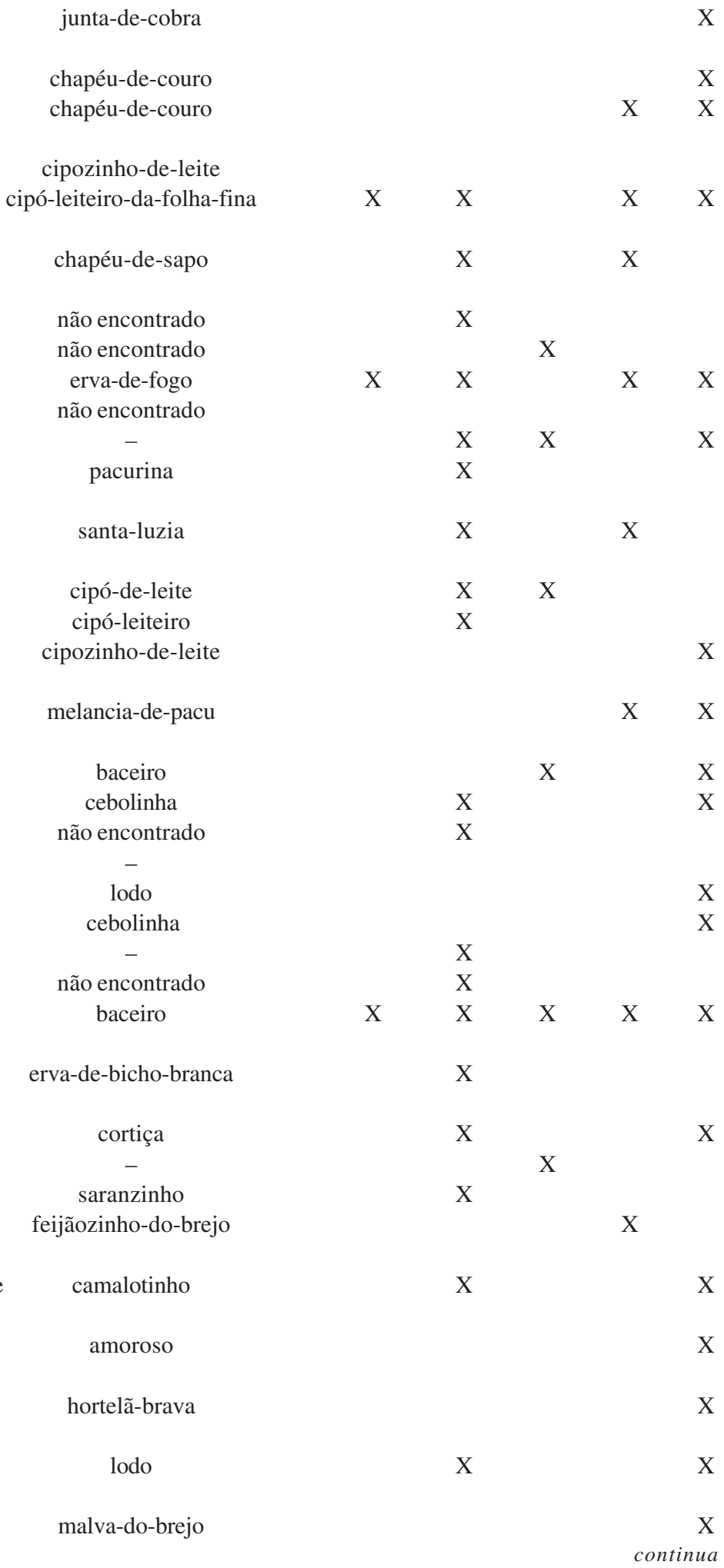


Tabela 1 (continuação)

\begin{tabular}{|c|c|c|c|c|c|c|}
\hline \multirow[t]{2}{*}{ Família/Espécie } & \multirow[t]{2}{*}{ Nome vulgar } & \multicolumn{5}{|c|}{ Baceiro } \\
\hline & & 1 & 2 & 3 & 4 & 5 \\
\hline \multicolumn{7}{|l|}{ ONAGRACEAE } \\
\hline Ludwigia grandiflora (Michx.) Greuter \& Burdet & florzeiro & & $\mathrm{X}$ & & & \\
\hline L. helminthorrhiza (Mart.) H. Hara & lombrigueira & & $X$ & $\mathrm{X}$ & $\mathrm{X}$ & \\
\hline L. leptocarpa (Nutt.) H. Hara & florzeiro & $\mathrm{X}$ & $\mathrm{X}$ & $\mathrm{X}$ & $\mathrm{X}$ & $\mathrm{X}$ \\
\hline L. nervosa (Poir.) H. Hara & lombrigueira & & $\mathrm{X}$ & & $\mathrm{X}$ & $\mathrm{X}$ \\
\hline L. tomentosa (Cambess.) H. Hara & florzeiro & & & & & $\mathrm{X}$ \\
\hline \multicolumn{7}{|l|}{ PLANTAGINACEAE } \\
\hline Bacopa monnierioides (Cham.) B.L. Rob. & vique & & & & & $\mathrm{X}$ \\
\hline B. salzmannii (Benth.) Wettst. ex Edwall & não encontrado & & & & & $\mathrm{X}$ \\
\hline B. stricta (Schrad.) Edwall & não encontrado & & & & & $\mathrm{X}$ \\
\hline \multicolumn{7}{|l|}{ POACEAE } \\
\hline Andropogon bicornis $\mathrm{L}$. & rabo-de-burro & $\mathrm{X}$ & & & & $\mathrm{X}$ \\
\hline Eragrostis hypnoides (Lam.) Britton, Sterns \& Poggenb. & não encontrado & & $X$ & & & \\
\hline Hymenachne amplexicaulis (Rudge) Nees & capim-de-capivara & & & $\mathrm{X}$ & & \\
\hline Imperata tenuis Hack. & sapé-fino & & & & & $\mathrm{X}$ \\
\hline Leersia hexandra $\mathrm{Sw}$. & felpudinho & & & & & $\mathrm{X}$ \\
\hline Luziola bahiensis (Steud.) Hitchc. & capim-do-brejo & & & & & $\mathrm{X}$ \\
\hline Panicum elephantipes Nees ex Trin. & capim-camalote & & $\mathrm{X}$ & & $\mathrm{X}$ & \\
\hline P. laxum Sw. & grama-do-carandazal & & & & & \\
\hline Paspalum repens P.J. Bergius & capim-fofo & & & & $X$ & \\
\hline \multicolumn{7}{|l|}{ POLYGONACEAE } \\
\hline Polygonum acuminatum Kunth & erva-de-bicho & $\mathrm{X}$ & $X$ & $\mathrm{X}$ & & \\
\hline P. ferrugineum Wedd. & fumo-bravo & & $X$ & $\mathrm{X}$ & & \\
\hline P. hispidum Kunth & erva-de-bicho & & $\mathrm{X}$ & & & \\
\hline P. punctatum Elliott & erva-de-bicho & & $\mathrm{X}$ & & & $\mathrm{X}$ \\
\hline \multicolumn{7}{|l|}{ PONTEDERIACEAE } \\
\hline Eichhornia azurea (Sw.) Kunth & camalote & & $\mathrm{X}$ & & $\mathrm{X}$ & \\
\hline E. crassipes (Mart.) Solms & camalote & & $\mathrm{X}$ & & $\mathrm{X}$ & \\
\hline Pontederia parviflora Alexander & guapé & & & & & $\mathrm{X}$ \\
\hline \multicolumn{7}{|l|}{ PTERIDACEAE } \\
\hline Ceratopteris pteridoides (Hook.) Hieron. & pé-de-sapo & & & & $\mathrm{X}$ & \\
\hline Pityrogramma calomelanos (L.) Link & samambaia-do-brejo & & & & & $\mathrm{X}$ \\
\hline \multicolumn{7}{|l|}{ URTICACEAE } \\
\hline Cecropia pachystachya Trec. & embaúba & & $\mathrm{X}$ & & & $\mathrm{X}$ \\
\hline \multicolumn{7}{|l|}{ VERBENACEAE } \\
\hline Lippia alba (Mill.) N.E. Br. & cidreira-do-campo & & $\mathrm{X}$ & & & \\
\hline \multicolumn{7}{|l|}{ VITACEAE } \\
\hline Cissus spinosa Cambess. & cipó-de-arraia & & $X$ & & $\mathrm{X}$ & $\mathrm{X}$ \\
\hline \multicolumn{7}{|l|}{ XYRIDACEAE } \\
\hline Xyris jupicai Rich. & cabeçudinho & & & & & $\mathrm{X}$ \\
\hline
\end{tabular}

observações (seca do local onde esse se encontra, substrato parcialmente vivo e histossolo menos espesso), que esta comunidade é a menos avançada dentre as estudadas.

A confecção do diagrama de Cluster, utilizando correlação de Pearson obtido por matriz de presença/ ausência de espécies (Fig. 2), relativo à composição florística das áreas (baceiros) estudadas, elucidou as diferenças entre estágios sucessionais mais e menos avançados. De acordo com essa figura, fica evidenciada a alta similaridade florística entre ilhas flutuantes em estágios sucessionais mais próximos. No caso, as mais avançadas, Baceiros 2 e 5 , se relacionam com maior proximidade, definindo um grupo bem delimitado. Essas duas comunidades também foram as mais ricas com relação à diversidade de espécies.

No extremo oposto, observou-se a menor relação de similaridade (maior distância) entre os Baceiros $3 \mathrm{e}$ 5, evidenciando a diferença na composição de espécies entre ilhas flutuantes em estágios distintos de sucessão, nesse caso, entre a menos e a mais avançada, dentre as estudadas. Observa-se também a grande diferença 


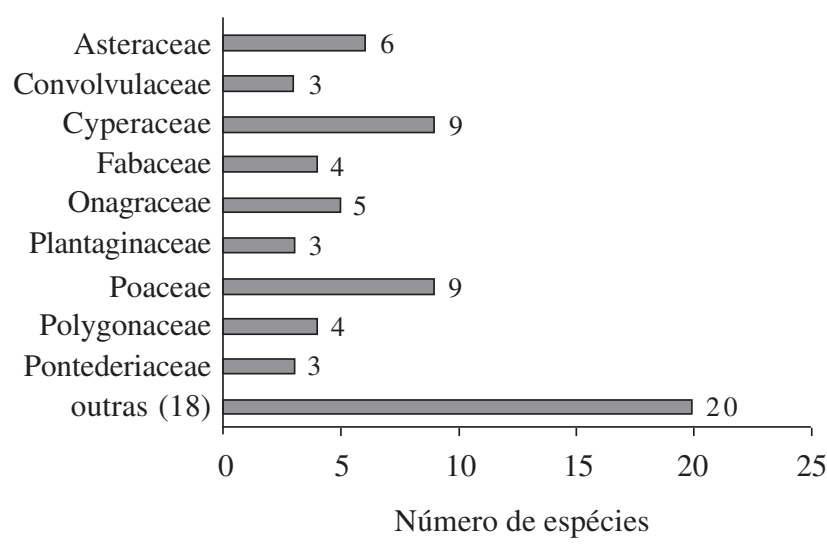

Figura 1. Famílias de macrófitas aquáticas epífitas mais representadas em cinco baceiros localizados no Pantanal, sub-regiões do Abobral e Miranda, município de Corumbá, MS, Brasil.

com relação ao número de espécies entre essas duas. Apesar do Baceiro 1 apresentar o menor número de espécies, este se encontra em estágio mais avançado que o Baceiro 3, uma vez que o substrato flutuante do primeiro é composto apenas por matéria orgânica em decomposição (além de mais espesso, com a substituição completa das macrófitas flutuantes livres) e pelo local onde se encontra apresentar maior permanência da coluna d'água.

As relações de similaridade florística entre os Baceiros 1, 3 e 4 se mostraram próximas entre si como ilustrado na Fig. 2, formando um grupo distinto dos Baceiros 2 e 5, além de apresentarem riquezas de espécies inferiores a estes. Dentro desse agrupamento, caracteriza-se ainda uma maior proximidade e conseqüentemente maior similaridade florística entre os Baceiros 1 e 4 . O índice de Sørensen (1948), aplicado aos Baceiros 1, 3 e 4 (Fig. 3), elucida que os Baceiros 1 e 4 apresentam $40 \%$ de similaridade florística, indicando através da composição de espécies, a presença em estágios intermediários de sucessão, e ainda que, entre os Baceiros 3 e 4 existe baixa taxa de similaridade (29\%). Apenas três espécies são encon-

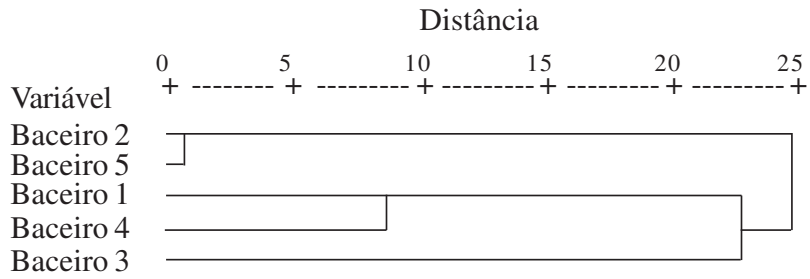

Figura 2. Dendrograma ilustrando a similaridade florística, obtido por matriz de presença $\times$ ausência das espécies presentes em cinco baceiros estudados no Pantanal, sub-regiões do Abobral e Miranda, município de Corumbá, MS, Brasil, baseado na distância euclidiana. tradas simultaneamente nessas três comunidades.

A distância em que se encontram os baceiros e as áreas destes não influenciaram na similaridade florística como poderia se esperar, ou seja, mais similares caso fossem próximos (devido aos mecanismos de dispersão) e de áreas equivalentes. Desse modo, os Baceiros 2 e 5 encontram-se a aproximadamente $28 \mathrm{~km}$ de distância e apresentam respectivamente, cerca de $40 \mathrm{~m}^{2}$ e $300 \mathrm{~m}^{2}$, portanto, destoando consideravelmente com relação a esses parâmetros. Tal fato ocorre semelhantemente entre os Baceiros 1 e 4, os quais se encontram relativamente distantes (cerca de $5,5 \mathrm{~km}$ ) e com áreas de aproximadamente $70 \mathrm{~m}^{2}$ e $280 \mathrm{~m}^{2}$. Em ambos os casos, apesar das diferenças mencionadas, foram observadas consideráveis similaridades florísticas. Por outro lado, a proximidade $(200 \mathrm{~m})$ dos Baceiros 2 e 3 não influenciou na similaridade da composição de espécies dessas duas comunidades. Diante de tais evidências, comprova-se que a composição florística das ilhas flutuantes é determinada prioritariamente pelo estágio de sucessão natural em que estas se encontram.

O esquema sucessional (Fig. 4), observado por Pott \& Pott (2003), demonstra a importância da permanência de água para a sobrevivência do baceiro, pois caso ocorra constante contato com a água, a ilha flutuante mantém-se em processo de sucessão natural acumulando matéria orgânica ao histossolo, vindo a morrer se houver seca no local. Este trabalho confirma

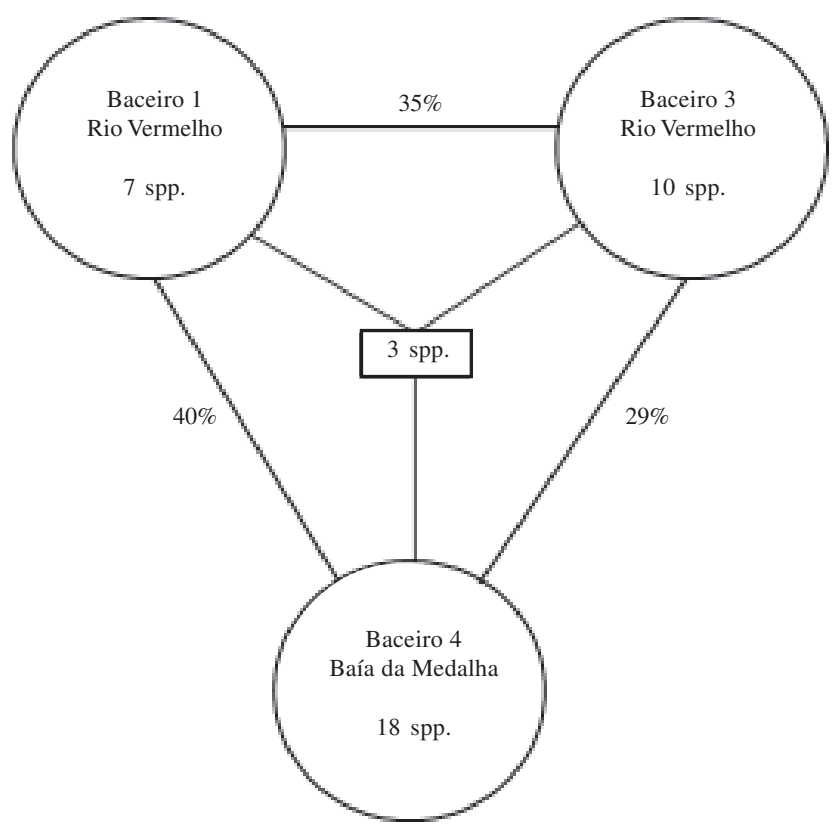

Figura 3. Diagrama ilustrando o índice de similaridade de SØrensen entre três baceiros estudados no Pantanal, sub-região de Miranda, município de Corumbá, MS, Brasil. 


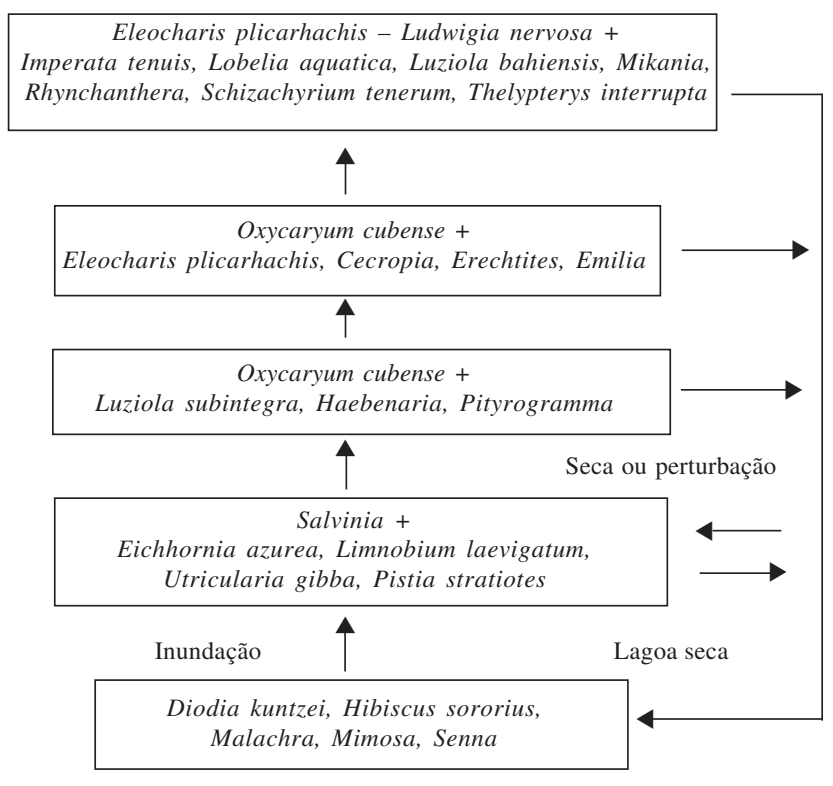

Figura 4. Esquema da sucessão natural da vegetação aquática, culminando em baceiros, modificado de Pott \& Pott (2003).

o esquema descrito por esses autores, já que o Baceiro 5, o qual apresenta maior tempo de sucessão, encontra-se no corpo d'água mais profundo, sendo portanto, o local que manteve por mais tempo a presença de água desde o surgimento dessa vegetação, possibilitando seu desenvolvimento ininterrupto e elevada espessura do histossolo.

As espécies de macrófitas aquáticas epífitas distribuídas nos baceiros caracterizam-se por apresentar constantemente contato com a água através de suas raízes. Nesse sentido, constituem esse tipo de vegetação espécies tolerantes ao estresse de alagamento (Scremin-Dias et al. 1999), sendo encontradas comumente em regiões constantemente alagadas ou temporariamente alagáveis. Enquadramse nesse perfil especialmente macrófitas aquáticas das formas biológicas anfíbia e emergente, por ocorrerem em locais que compartilham fatores comuns às condições presentes no solo dos baceiros.

Entretanto, foram amostradas espécies de formas biológicas distintas que se mostraram adaptadas às condições do histossolo, ocorrendo enraizadas neste. Assim, Ceratopteris pteridoides, Eichhornia crassipes, Limnobium laevigatum e Utricularia gibba, que normalmente são flutuantes livres, e Eichhornia azurea, flutuante fixa, podem ser consideradas "epífitas casuais" (Tab. 2).

Tabela 2. Macrófitas aquáticas "não epífitas" encontradas em meio ao solo orgânico de baceiros e "epífitas casuais", seguidas de suas respectivas formas biológicas, no Pantanal, sub-regiões do Abobral e Miranda, município de Corumbá - MS. N. Ep. = não epífita; Ep. C. = epífita casual.

\begin{tabular}{|c|c|c|c|}
\hline Família/Espécie & Nome vulgar & Forma biológica & Ocorrência \\
\hline \multicolumn{4}{|l|}{ ARACEAE } \\
\hline Pistia stratiotes $\mathrm{L}$. & alface-d’água & flutuante livre & N. Ep. \\
\hline \multicolumn{4}{|l|}{ AZOLLACEAE } \\
\hline Azolla filiculoides Lam. & azola & flutuante livre & N. Ep. \\
\hline \multicolumn{4}{|l|}{ САВОМВАСЕАЕ } \\
\hline Cabomba furcata Schult. \& Schult. f. & lodo & submersa fixa & N. Ep. \\
\hline \multicolumn{4}{|l|}{ HYDROCHARITACEAE } \\
\hline Limnobium laevigatum (Humb. \& Bonpl. ex Willd.) Heine & camalotinho & flutuante livre & Ep. C. \\
\hline \multicolumn{4}{|l|}{ LENTIBULARIACEAE } \\
\hline Utricularia gibba L. & lodo & flutuante livre & Ep. C. \\
\hline \multicolumn{4}{|l|}{ LIMNOCHARITACEAE } \\
\hline Hydrocleys nymphoides (Willd.) Buch. & lagartixa & flutuante fixa & N. Ep. \\
\hline \multicolumn{4}{|l|}{ NYMPHAEACEAE } \\
\hline Nymphaea amazonum Mart. \& Zucc. & lagartixa & flutuante fixa & N. Ep. \\
\hline \multicolumn{4}{|l|}{ PARKERIACEAE } \\
\hline Ceratopteris pteridoides (Hook.) Hieron. & pé-de-sapo & flutuante livre & Ep. C. \\
\hline \multicolumn{4}{|l|}{ PONTEDERIACEAE } \\
\hline Eichhornia azurea (Sw.) Kunth & camalote & flutuante fixa & Ep. C. \\
\hline E. crassipes (Mart.) Solms & camalote & flutuante livre & Ep. C. \\
\hline \multicolumn{4}{|l|}{ PHYLLANTHACEAE } \\
\hline Phyllanthus fluitans Benth. ex Müll. Arg. & orelha-de-onça & flutuante livre & N. Ep. \\
\hline \multicolumn{4}{|l|}{ SALVINIACEAE } \\
\hline Salvinia auriculata Aubl. & orelha-de-onça & flutuante livre & N. Ep. \\
\hline S. minima Baker & orelha-de-onça & flutuante livre & N. Ep. \\
\hline
\end{tabular}




\section{Agradecimentos}

À Fundação O Boticário, por arcar com os custos do projeto através do Programa de Incentivo à Conservação da Natureza; à PROPP/UFMS, pelo apoio financeiro e hospedagem; a Samuel Boff, pela ajuda nos trabalhos de campo.

\section{Referências bibliográficas}

Allem, A.C. \& Valls, J.F. 1987. Recursos forrageiros nativos do Pantanal mato-grossense. Brasília, Anais EMBRAPACENARGEN. Documentos, 8.

Amaral Filho, Z.P. 1986. Solos do Pantanal mato-grossense. Simpósio sobre Recursos Naturais e Sócio-econômicos do Pantanal. Pp. 91-103. In: Anais CPAP/EMBRAPA. ed. Brasília.

APG (Angiosperm Phylogeny Group) II. 2003. An update of the Angiosperm Phylogeny Group classification for the orders and families of flowering plants: APG II. Journal of the Linnean Society 141: 399-436.

BRASIL. Ministério do Interior. 1979. Estudo de desenvolvimento integrado da Bacia do Alto Paraguai (EDIBAP). Relatório da $\mathbf{1}^{\text {a }}$ fase - Descrição Física e Recursos Naturais. Tomo II. Brasília.

Brummitt, R.K. \& Powell, C.E. 1992. Authors of plant names. Kew, Royal Botanic Garden.

Cook, C.D.K. 1974. Water plants of the world. The Hague, W. Junk.

Cuadrado, G.A. \& Neiff, J.J. 1993. Palynology of embalsados in distrophic lakes in Northeastern of Argentina. Revista Brasileira de Biologia 53: 443-451.

Esteves, F.A. 1998. Fundamentos de Limnologia. $2^{\text {a }}$ ed. Rio de Janeiro, Interciência.

Irgang, B.E.; Pedralli, G. \& Waechter, J.I. 1984. Macrófitos aquáticos da Estação Ecológica do Taim, Rio Grande do sul, Brasil. Roessleria 6: 395-404.

Junk, W.J. \& Silva, C.J. 1999. O conceito do pulso de inundação e suas implicações para o Pantanal de Mato Grosso. Pp. 17-28. In: Anais do II Simpósio sobre Recursos Naturais e Sócio-Econômicos do Pantanal. Manejo e Conservação. Corumbá, EMBRAPA-DDT.

Köppen, W. 1931. Climatologia. Buenos Aires, Fondo de Cultura Económica.

Kramer, K.U. \& Green P.S. 1990. The families and genera of vascular plants. Pteridophytes and Gymnosperms. v. I. New York, Springer Verlag.
Lima, Z.M.; Paula, A.M.; Sérgio, E.C.; Soares, C.R. \& Macedo, M. 1999. Aspectos ecológicos da dispersão em "camalotes" de macrófitas aquáticas na Baía Piuvial, Pantanal de Poconé - MT. Pp. 381-385. In: Anais do II Simpósio sobre Recursos Naturais e Sócio-Econômicos do Pantanal. Manejo e Conservação. Corumbá, EMBRAPA-DDT.

Martins, H.F. \& Carauta, J.P.P. 1984. Plantas aquáticas. Classificação e comentários. Atas da Sociedade Botânica do Brasil 2: 101-104.

Neiff, J.J. 1982. Esquema sucesional de la vegetacion en islas flotantes del chaco argentino. Boletin de la Sociedad Argentina de Botánica 21: 325-341.

Pedralli, G. 2000. Padrões florísticos como subsídios à conservação da biodiversidade de macrófitas aquáticas. Tópicos Atuais em Botânica, Brasília, EMBRAPA.

Pott, V.J. \& Pott, A. 2000. Plantas aquáticas do Pantanal. 1ed. Corumbá, EMBRAPA.

Pott, V.J. \& Pott, A. 2003. Dinâmica da vegetação aquática do Pantanal. Pp. 145-162. In: S.M. Thomaz \& L.M. Bini. Ecologia e manejo de macrófitas aquáticas. Maringá, Editora da Universidade Estadual de Maringá.

Risser, P.G. 1995. The status of the science examining ecotones. A dynamic aspect of landscape in the area of steep gradients between more homogeneous vegetation associations. BioScience 45: 318-325.

Scremin-Dias, E.; Pott, V.J.; Hora, R.C. \& Souza, P.R. 1999. Nos jardins submersos da Bodoquena: guia para identificação das plantas aquáticas de Bonito e região. Campo Grande, MS, Ed. da UFMS.

Silva, C.J. 1984. Nota prévia sobre o significado biológico dos termos usados no Pantanal Mato-grossense, "Batume" e "Diquada". Revista Universidade Federal de Mato Grosso 4: 30-36.

Silva, J.S.V. \& Abdon, M.M. 1998. Delimitação do Pantanal Brasileiro e suas sub-regiões. Pesquisa Agropecuária Brasileira 33(Número Especial): 1703-1711.

Sørensen, T.A. 1948. A method for establishing groups of equal magnitud in plant sociology based on similarity of species content, and its application to analyses of the vegetation on Danish commons. Kongelige Danske Videnskabernes Selskab 5: 1-34.

Tur, N.M. 1965. Un caso de epifitismo acuático. Boletin de la Sociedad Argentina de Botánica 10: 323-327.

Tur, N.M. 1972. Embalsados y camalotes de la Región Isleña Del Paraná Médio. Darwiniana 17: 397-407.

Wilhelmy, H. 1957. Das grosse Pantanal in Mato Grosso. Deutscher Geographentag, Wuerzburg. Pp. 45-71. In: Tagunsberichte und wissenchaftlichen abhandlungen. Wiesbaden, Franz Steiner. 